\title{
Passive-microwave remote sensing of snow
}

\author{
Tosio KoIKe, \\ Department of Civil Engineering, Nagaoka University of Technology, Nagaoka, Niigata 940-21, Japan \\ TOMOYUKi SuHAMA \\ System Engineering Division, PASCO Corporation, Higasiyama, Meguro, Tokyo 153, Japan
}

\begin{abstract}
The radiative-transfer theory and the Rayleigh scattering model were used to obtain the microwave brightness temperatures from dry snowpacks on the ground, which was modelled by a scattering dielectric layer and an underlying homogeneous half-space. The total radio brightness at the radiometer was the sum of the direct and diffuse radiation field intensities. The result of the model application was in good agreement with the observational data, which were obtained through the airborne experiments for the MOS-1 Verification Program around Asahikawa, Hokkaido, Japan, in February 1988.
\end{abstract}

\section{INTRODUCTION}

Due to the dynamic behavior of snow and ice, efficient monitoring is possible only by using remote-sensing methods which provide synoptic coverage over large areas. Microwave techniques offer important advantages for snow mapping: the all-weather capability and the possibility to detect snow water equivalent and other parameters of the snow volume.

First snow studies with spaceborne passive-microwave data were based on the Electrically Scanning Microwave Radiometer (ESMR) of Nimbus-5, which operated at $19 \mathrm{GHz}$ (Foster and others, 1980). The five-frequency $(6.6,10.7,18,21,37 \mathrm{GHz})$ dual-polarized Scanning Multichannel Microwave Radiometer (SMMR), which was launched on Nimbus 7 in 1978, provided the improved spectral capability (Chang, 1986). A similar system which had an additional channel at $85 \mathrm{GHz}$, Special Sensor Microwave Imager (SSM/I), was launched in 1987 on the US Air Force Defense Meteorological Satellite Program (DMSP) satellite. In the same year the Microwave Scanning Radiometer (MSR), which was a Dicke type radiometer at $23.8 \mathrm{GHz}$ and $31.4 \mathrm{GHz}$, was launched on the Marine Observation Satellite 1 (MOS-1).

Synchronizing the airborne-MSR experiments on passing days of MOS-1, ground-truth data were obtained at the center of Hokkaido in February 1988. On the basis of these data, both theoretical and experimental studies on the passive-microwave remote sensing of snow are presented in this paper.

\section{MICROWAVE EMISSION FROM SNOWPACK}

Microwaves are able to penetrate snowpack and provide information on properties of the snow volume (Rott,
1987). If the wavelength is large compared to the size of the snow grains, the amount of penetration can be described by the bulk dielectric constant. The presence of liquid water strongly increases the dielectric losses. Therefore, wet snow is opaque for microwaves and the emitted signal originates from a very thin layer at the top of the snowpack. Wet snow has a high emissivity throughout the microwave region. Therefore permanently wet snowcovers can be hardly discriminated from snow-free ground by microwave radiometry. In dry snow, scattering effects are more pronounced at shorter wavelengths (Chang and others, 1987) and depend not only on the complex dielectric constant of the scatterers but also on their size and shape. Larger grain sizes within the snowpack cause more scattering of microwave radiation as the grain size approaches the wavelength (Chang and others, 1976; Matzler and others, 1982; Hall and others, 1986; Hall 1987). Due to volume scattering the emissivity of dry snow is clearly reduced at frequencies above $15 \mathrm{GHz}$. The decrease of emissivity not only depends on the total amount of snow on the ground, but is strongly affected by the grain-size (Rott, 1987).

The radiative-transfer theory and a Rayleigh scattering model are used to obtain the microwave temperature (England, 1975). The theory based upon a scattering dielectric layer of average thickness, $D$, over a homogeneous half-space is applied to emission from a snowpack and underlying soil as shown in Figure 1. The surface and interface, while quasi-specular, are sufficiently irregular with respect to microwave wavelengths that over the field of view of the radiometer the interference phenomena are effectively averaged. The microwave brightness temperatures observed by a radiometer are sought in two parts as shown in Figure 2: the direct field consisting of reflectedsky radiation and thermal radio emission from the snowpack and soil; and the diffuse field resulting from radiation scattered from the direct field. 


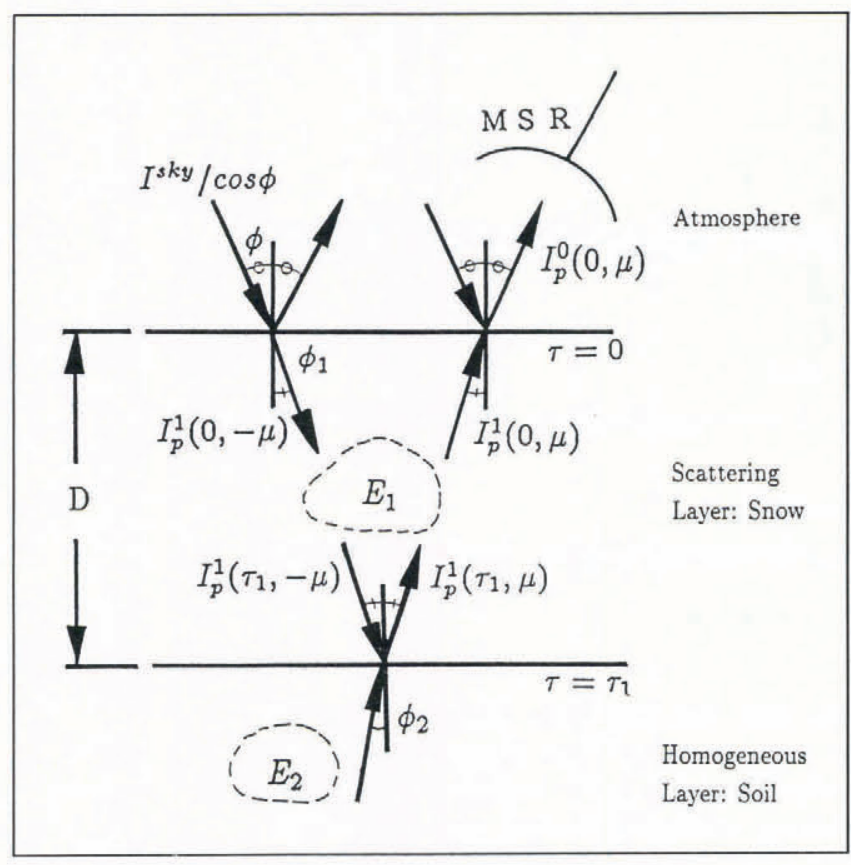

Fig. 1. Snowpack model.

The total radio brightness at the radiometer, $T B_{p}(\mu)$, is the sum of the direct component, $T B_{p}(\mu)_{\mathrm{dir}}$, and the diffuse one, $T B_{p}(\mu)_{\text {dif }}$, i.e.,

$$
T B_{p}(\mu)=T B_{p}(\mu)_{\mathrm{dir}}+T B_{p}(\mu)_{\mathrm{dif}} .
$$

The nodal equations at the surface and the interface for the direct intensities $I_{p}^{n}(\tau, \mu)$, where $n$ denotes the medium (0: air, 1: snow, 2: soil), $p$ is polarization, $\tau$ is optical depth, and $\mu$ is direction cosine of the ray within the layer, are

$$
\begin{aligned}
I_{p}^{0}(0, \mu)-\frac{1-R_{p}^{1}(\mu)}{\varepsilon_{1}} I_{p}^{1}(0, \mu) & =R_{p}^{1}(\mu) \frac{I^{\mathrm{sky}}}{\cos \phi}, \\
I_{p}^{1}(0,-\mu)-R_{p}^{1}(\mu) I_{p}^{1}(0, \mu) & =\varepsilon_{1}\left(1-R_{p}^{1}(\mu)\right) \frac{I^{\mathrm{sky}}}{\cos \phi}, \\
I_{p}^{1}(0, \mu)-I_{p}^{1}\left(\tau_{1}, \mu\right) e^{-\tau_{1} / \mu} & =E_{1}\left(\int_{0}^{\tau_{1}} \mu\right), \\
I_{p}^{1}\left(\tau_{1},-\mu\right)-I_{p}^{1}(0,-\mu) e^{-\tau_{1} / \mu} & =E_{1}\left({ }_{\tau_{1}}^{0}-\mu\right), \\
I_{p}^{1}\left(\tau_{1}, \mu\right)-R_{p}^{2}(\mu) I_{p}^{1}\left(\tau_{1},-\mu\right) & =\frac{\varepsilon_{1}}{\varepsilon_{2}}\left(1-R_{p}^{2}(\mu)\right) E_{2},
\end{aligned}
$$

where $R_{p}^{1}$ and $R_{p}^{2}$ are the Fresnel reflection coefficients at the surface and interface, respectively, $\varepsilon_{n}$ is the relative permittivity of the medium $n$, and $E_{n}\left({ }_{a}^{b} \mu\right)$ is the intensity within medium $n$ caused by thermal emission between depths $b$ and $a$ in direction $\mu$. That is,

$$
\begin{aligned}
E_{1}\left(\begin{array}{c}
\tau_{1} \\
0
\end{array}\right) & =\int_{0}^{\tau^{\eta}} \frac{\beta_{1} \varepsilon_{1} C T_{1}}{\alpha_{1}} e^{-\tau / \mu} \frac{1}{\mu} \mathrm{d} r \\
& =\frac{\beta_{1} \varepsilon_{1} C T_{1}}{\alpha_{1}}\left(1-e^{-\tau_{1} / \mu}\right) \\
& =E_{1}\left({ }_{\tau_{1}}^{0}-\mu\right), \\
E_{2} & =\varepsilon_{2} C T_{2},
\end{aligned}
$$

where the constant $C$ derives from the Rayleigh-Jeans approximation to the Plank emission. The brightness temperature of the direct field sensed at the radiometer is

$$
T B_{p}(\mu)_{\mathrm{dir}}=I_{p}^{0}(0, \mu) / C .
$$

The intensity of the diffuse radiation, $G_{p}\left(\tau, \mu^{\prime}\right)$, is reduced by total absorption and increased by radiation scattered from the direct and diffuse field. That is, the radiative transfer equations are

$$
\begin{aligned}
\mu \frac{\partial G_{V}(\tau, \mu)}{\partial \tau} & =G_{V}(\tau, \mu) \\
& -\frac{\omega_{0}}{2} \int_{-1}^{+1} P_{V V}\left(\mu, \mu^{\prime}\right)\left[G_{V}\left(\tau, \mu^{\prime}\right)+I_{V}\left(\tau, \mu^{\prime}\right)\right] \\
& +P_{V H}\left(\mu, \mu^{\prime}\right)\left[G_{H}\left(\tau, \mu^{\prime}\right)+I_{H}\left(\tau, \mu^{\prime}\right)\right] \mathrm{d} \mu^{\prime},(10) \\
\mu \frac{\partial G_{H}(\tau, \mu)}{\partial \tau} & =G_{H}(\tau, \mu) \\
& -\frac{\omega_{0}}{2} \int_{-1}^{+1} P_{H V}\left(\mu, \mu^{\prime}\right)\left[G_{V}\left(\tau, \mu^{\prime}\right)+I_{V}\left(\tau, \mu^{\prime}\right)\right] \\
& +P_{H H}\left(\mu, \mu^{\prime}\right)\left[G_{H}\left(\tau, \mu^{\prime}\right)+I_{H}\left(\tau, \mu^{\prime}\right)\right] \mathrm{d} \mu^{\prime},(11)
\end{aligned}
$$

where $H$ and $V$ show the horizontal and vertical polarization, respectively, $\omega_{0}$ is the scattering albedo, and $P_{p q}\left(\mu, \mu^{\prime}\right)$ is the normalized Rayleigh scattering phase function for the rotationally symmetric case; $\operatorname{Ppq}\left(\mu, \mu^{\prime}\right)$ relates the $p$ polarized radiation scattered in any direction $\mu$ to the incident field of polarization $q$ from all azimuths about $z$ having direction $\cos \mu^{\prime}$. From Chandrasekhar (1960),

$$
\begin{aligned}
& P_{V V}\left(\mu, \mu^{\prime}\right)=\frac{3}{4}\left[2\left(1-\mu^{2}\right)\left(1-\mu^{\prime} 2\right)+\mu^{2} \mu^{\prime} 2\right], \\
& P_{V H}\left(\mu, \mu^{\prime}\right)=\frac{3}{4} \mu^{2}, \\
& P_{H V}\left(\mu, \mu^{\prime}\right)=\frac{3}{4} \mu^{\prime} 2, \\
& P_{H H}\left(\mu, \mu^{\prime}\right)=\frac{3}{4} .
\end{aligned}
$$
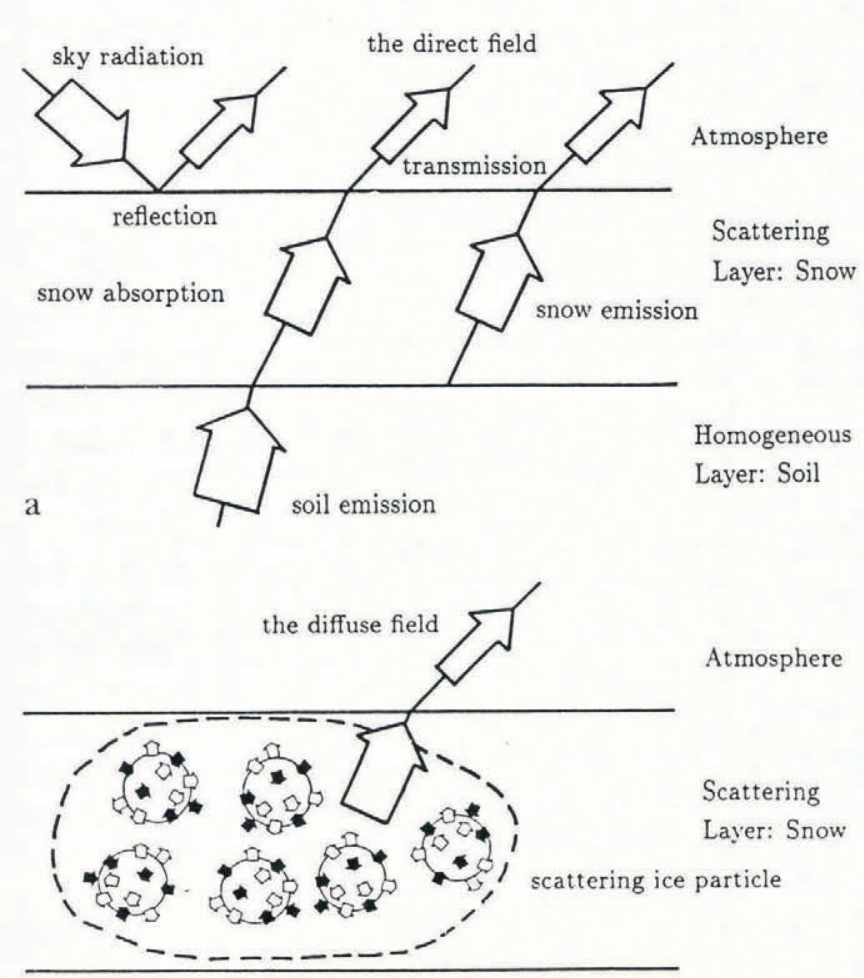

b

Homogeriecus Layer: Soil

Fig. 2. $a$, the direct field; $b$, the diffuse field. 


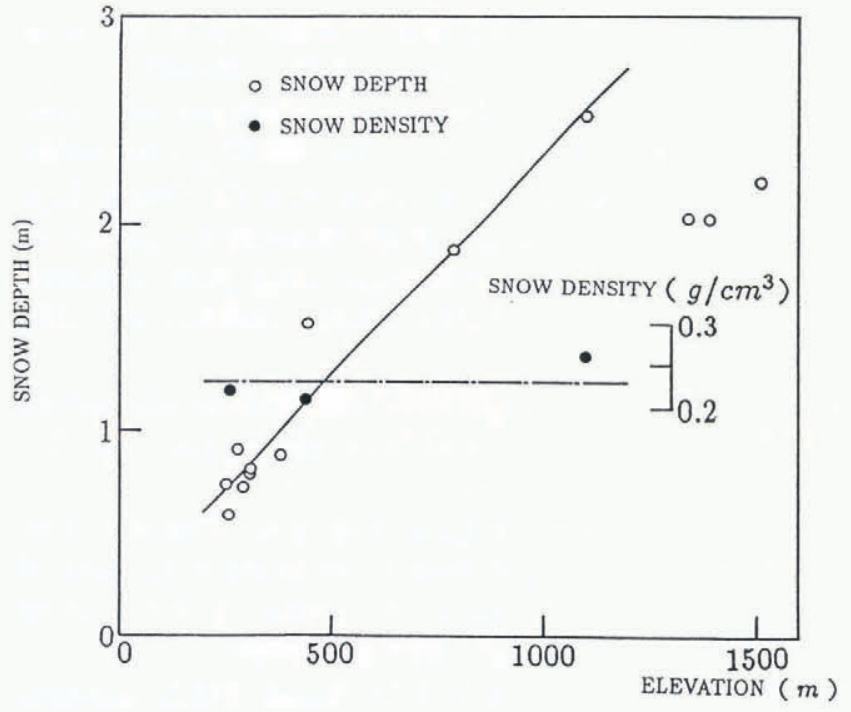

Fig. 3. Altitudinal distribution of snow depth and density.

An approximate solution to Equations (10) and (11) can be obtained through replacement of the integral by an $n$ th-order Gaussian quadrature.

\section{MODEL APPLICATION}

National Space Development Agency of Japan (NASDA) planned and initiated the MOS-1 Verification Program (MVP) to evaluate the MOS-1 observation system. As a part of MVP, NASDA conducted airborne experiments on passing days of MOS-1 in February 1988. Synchro- nous with the airborne experiments, ground truth data, i.e. snow depth, snow density, and temperature profile of snowpack, were obtained along the route of the aircraft from Mount Asahidake to Biei on Hokkaido. Figure 3 shows the altitudinal distribution of snow depth and density. The distribution of snow depth is well expressed by a linear function of the elevation, except around the top of the mountain where snow densities are approximately constant. Therefore the altitudinal distribution of snow water equivalent is expressed by a linear function. The snow was dry over all the experiment area.

Microwave brightness temperature depends on the snow grain-size, which was not obtained, as well as the total mass and temperature profile of the snowpack. Therefore, assuming several values of snow grain-size, the brightness temperature at each snow-observation site is calculated by using the radiative-transfer model. Figure 4 shows the comparison of the calculated radiance and that observed by the airborne MSR. The calculated brightness temperatures agree with the aircraft observation data quite well for the optimum values of grain-size at each frequency: $0.6 \mathrm{~mm}$ at $23 \mathrm{GHz}$ and $0.4 \mathrm{~mm}$ at $31 \mathrm{GHz}$. These model results indicate that the grain-size of a snowpack is a major factor in determination of the microwave brightness temperature. It is presumed that the different optimum values of grain-size are caused from the Rayleigh scattering assumption.

\section{CONCLUSION}

The preliminary results show that passive-microwave data have the potential to provide useful information
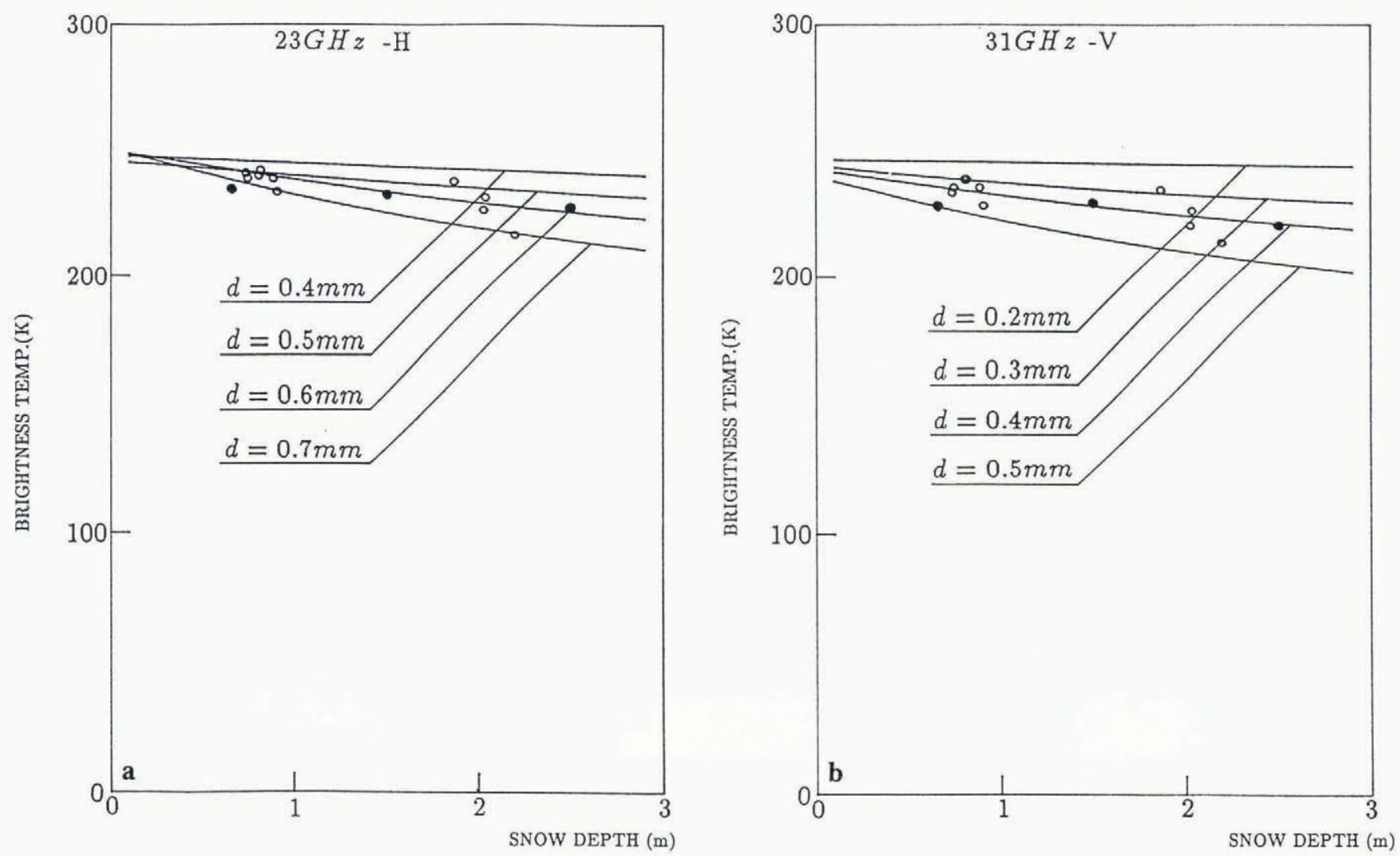

Fig. 4. Calculated brightness temperature vs airborne-MSR observed brightness temperature. $a, 23 G H z-H ; b, 31 G H z-$ V. 
related to dry-snowpack properties. A significant relationship between snow water equivalent and microwave brightness temperature can be derived by using radiativetransfer theory and a scattering model.

The main drawback of passive-microwave remote sensing from satellite is the spatial resolution. It limits the use of these data in the mountainous area. No significant improvements can be expected. However, several passivemicrowave sensors will be launched in the 1990s. Extensive research is needed to improve the algorithms for snow mapping using the information obtained from other types of remote sensors and the ground data.

\section{REFERENCES}

Chandrasekhar, S. 1960. Radiative transfer. New York, Dover.

Chang, A.T.C. 1986. Nimbus-7 SMMR snow cover data. In Kukla, G., R. G. Barry, A. D. Hecht and D. R. Wiesnet, eds. Snow Watch'85. Boulder, CO, University of Colorado. Cooperative Institute for Research in Environmental Sciences, 181-187.

Chang, A. T. C., P. Gloersen, T. Schmugge, T. T. Wilheit and H.J. Zwally. 1976. Microwave emission from snow and glacier ice. 7. Glaciol., 16(74), 23-39.

Chang, A. T. C., J.L. Foster and D. K. Hall. 1987.
Microwave snow signatures $(1.5 \mathrm{~mm}$ to $3 \mathrm{~cm}$ ) over Alaska. Cold Reg. Sci. Technol., 13(2), 153-160.

England, A.W. 1975. Thermal microwave emission from a scattering layer. F. Geophys. Res., 80(32), 4484-4496.

Foster, J. L., A. Rango, D. K. Hall, A. T. C. Chang, L. J. Allison and B. C. Diesen. 1980. Snowpack monitoring in North America and Eurasia using passive microwave satellite data. Remote Sensing Environ., 10, 185198.

Hall, D. K. 1987. Influence of depth hoar on microwave emission from snow in northern Alaska. Cold Reg. Sci. Technol., 13(3), 225-231.

Hall, D. K., A.T.C. Chang and J.L. Foster. 1986. Detection of the depth-hoar layer in the snow-pack of the Arctic coastal plain of Alaska, U.S.A., using satellite data 7 . Glaciol., 32(110), 87-94.

Mätzler, C., E. Schanda and W. Good. 1982. Towards the definition of optimum sensor specifications for microwave remote sensing of snow. IEEE Trans. Geosci. Remote Sensing, GE-20(1), 57-66.

Rott, H. 1987. Remote sensing of snow. International Association of Hydrological Sciences Publication 166 (Symposium at Vancouver 1987 - Large Scale Effects of Seasonal Snow Cover), 279-290.

The accuracy of references in the text and in this list is the responsibility of the authors, to whom queries should be addressed. 